\title{
Experimental and Computational Analysis of Para-Hydroxy Methylcinnamate following Photoexcitation
}

\author{
Jack Dalton, Gareth W. Richings (D), Jack M. Woolley, Temitope T. Abiola (D), Scott Habershon * \\ and Vasilios G. Stavros * \\ Department of Chemistry, University of Warwick, Coventry CV4 7AL, UK; J.Dalton.1@warwick.ac.uk (J.D.); \\ G.Richings@warwick.ac.uk (G.W.R.); Jack.Woolley@warwick.ac.uk (J.M.W.); \\ Temitope.Abiola@warwick.ac.uk (T.T.A.) \\ * Correspondence: S.Habershon@warwick.ac.uk (S.H.); V.Stavros@warwick.ac.uk (V.G.S.)
}

check for

updates

Citation: Dalton, J.; Richings, G.W.; Woolley, J.M.; Abiola, T.T.; Habershon, S.; Stavros, V.G. Experimental and

Computational Analysis of

Para-Hydroxy Methylcinnamate following Photoexcitation. Molecules 2021, 26, 7621. https://doi.org/ $10.3390 /$ molecules 26247621

Academic Editors: Tolga Karsili and Barbara Marchetti

Received: 26 November 2021 Accepted: 10 December 2021 Published: 15 December 2021

Publisher's Note: MDPI stays neutral with regard to jurisdictional claims in published maps and institutional affiliations.

Copyright: (c) 2021 by the authors. Licensee MDPI, Basel, Switzerland. This article is an open access article distributed under the terms and conditions of the Creative Commons Attribution (CC BY) license (https:// creativecommons.org/licenses/by/ $4.0 /)$.

\begin{abstract}
Para-hydroxy methylcinnamate is part of the cinnamate family of molecules. Experimental and computational studies have suggested conflicting non-radiative decay routes after photoexcitation to its $\mathrm{S}_{1}\left(\pi \pi^{*}\right)$ state. One non-radiative decay route involves intersystem crossing mediated by an optically dark singlet state, whilst the other involves direct intersystem crossing to a triplet state. Furthermore, irrespective of the decay mechanism, the lifetime of the initially populated $S_{1}\left(\pi \pi^{*}\right)$ state is yet to be accurately measured. In this study, we use time-resolved ion-yield and photoelectron spectroscopies to precisely determine the $S_{1}\left(\pi \pi^{*}\right)$ lifetime for the s-cis conformer of para-hydroxy methylcinnamate, combined with time-dependent density functional theory to determine the major non-radiative decay route. We find the $S_{1}\left(\pi \pi^{*}\right)$ state lifetime of s-cis para-hydroxy methylcinnamate to be $\sim 2.5$ picoseconds, and the major non-radiative decay route to follow the $\left[{ }^{1} \pi \pi^{*} \rightarrow{ }^{1} \mathrm{n} \pi^{*} \rightarrow{ }^{3} \pi \pi^{*} \rightarrow \mathrm{S}_{0}\right]$ pathway. These results also concur with previous photodynamical studies on structurally similar molecules, such as para-coumaric acid and methylcinnamate.
\end{abstract}

Keywords: ultrafast spectroscopy; DFT; gas-phase; VMI; photochemistry; photophysics; cinnamate

\section{Introduction}

Cinnamates and coumaric acids have been widely studied to elucidate their remarkable light absorbing properties, driven by efficient non-radiative decay (NRD) pathways [1-5]. In nature, these chromophores are embedded in proteins to enable vitally important processes to occur. A prime example is phototaxis in the photoactive yellow protein (PYP). PYP was first discovered in the Ectothiorhodospira halophila bacterium [6-8] and utilizes the photoisomerization of para-coumaric acid ( $p$-CA) to facilitate a negative phototactic response towards blue light, thus protecting the bacterium from potentially harmful photodamage [9-12]. Similar families of molecules are used in sunscreen formulations due to their photoprotective properties against potentially harmful ultraviolet A (UVA, 315-400 nm) and B (UVB, 280-315 $\mathrm{nm}$ ) radiation from the Sun [13-15].

Para-hydroxy methylcinnamate ( $p$-HMC; Figure $1 \mathrm{a}$ ) is the simplest ester derivative of $p$-CA and exhibits a strong absorption peak in the UVB region of the electromagnetic spectrum. Upon UV irradiation, the optically bright $S_{1}\left(\pi \pi^{*}\right)$ state is predominantly populated and undergoes relaxation via multiple NRD processes, returning to the electronic ground $S_{0}$ state. Although the decay dynamics of $p$-HMC following photoexcitation to the ${ }^{1} \pi \pi^{*}$ state are well-studied, there have been some conflicting conclusions regarding the dominant NRD route in the gas-phase. An early theory suggested that decay via the ${ }^{1} \pi \sigma^{*}$ dissociative state may dominate, analogous to what is observed in phenol [16]. The lowest lying ${ }^{1} \pi \sigma^{*}$ state in phenol is dissociative along the $\mathrm{O}-\mathrm{H}$ bond and ultimately leads to a conical intersection $(\mathrm{CI})$ with the $\mathrm{S}_{0}$ state, providing rapid relaxation of the molecule $[17,18]$. Upon deuteration of the $\mathrm{OH}$ moiety, a dramatic change in excited-state lifetime is observed, 
indicating that the excited-state decay in phenol is mediated via tunnelling $[19,20]$. In contrast, deuteration of the $\mathrm{OH}$ moiety in $p$-HMC results in very little change in excited-state lifetime, implying that $\mathrm{O}-\mathrm{H}$ fission is unavailable to $p$-HMC following photoexcitation to the $S_{1}$ state [21].
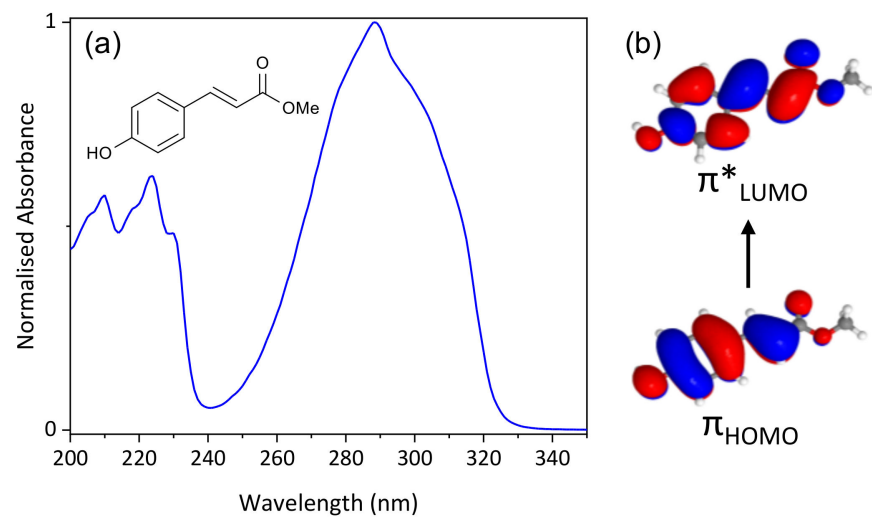

Figure 1. (a) UV absorption spectrum of para-hydroxy methylcinnamate ( $p$-HMC) (structure shown inset) in cyclohexane. (b) $p$-HMC's $\pi$ highest occupied molecular orbital $\left(\pi_{\mathrm{HOMO}}\right)$ and $\pi^{*}$ lowest unoccupied molecular orbital ( $\left.\pi^{*} \mathrm{LUMO}\right)$ relating to the $S_{1}\left(\pi \pi^{*}\right)$ state.

Following this observation, Smolarek et al. proposed an alternative NRD route for $p$-HMC, mirroring that of $p$-CA. This was based on the similarities in linewidths (and hence excited state lifetimes) of the photoexcitation spectra of $p$-HMC and $p$-CA, implying a comparable NRD route in both systems [21,22].

$p$-CA and methylcinnamate (MC), both structurally very similar to $p$-HMC, are photochemically and photo-physically well understood. It therefore stands to reason that they can be used as starting models for unravelling the NRD dynamics of $p$-HMC. Upon photoexcitation of $p$-CA to the ${ }^{1} \pi \pi^{*}$ state in the gas-phase, the NRD process shown in Figure 2a dominates, with the observed lifetime of the ${ }^{1} \pi \pi^{*}$ and ${ }^{3} \pi \pi^{*}$ states measured as $\leq 10$ picoseconds (ps) and $20 \pm 4$ nanoseconds (ns), respectively [5]. For MC, the same NRD process dominates with the ${ }^{1} \pi \pi^{*},{ }^{1} n \pi^{*}$ and ${ }^{3} \pi \pi^{*}$ states having been experimentally observed. The observed lifetimes are also remarkably similar to $p$-CA, being $4.5 \pm 1.1 \mathrm{ps}$ and $20 \mathrm{~ns}$ for the ${ }^{1} \pi \pi^{*}$ and ${ }^{3} \pi \pi^{*}$ states, respectively $[23,24]$.

(a)

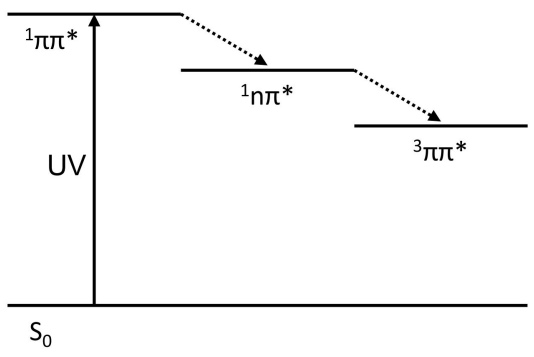

(b)

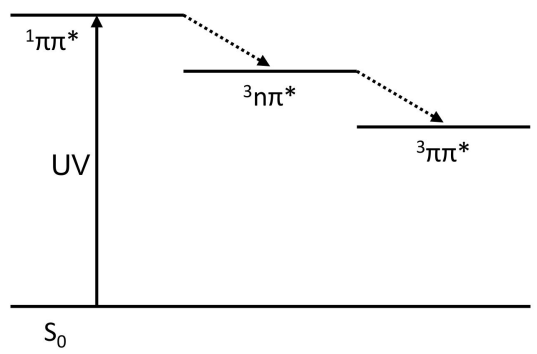

Figure 2. Possible non-radiative decay (NRD) routes following the absorption of UV light. (a) Internal conversion from the ${ }^{1} \pi \pi^{*}$ to the ${ }^{1} \mathrm{n} \pi^{*}$ state followed by intersystem crossing to the ${ }^{3} \pi \pi^{*}$ state. (b) Direct intersystem crossing from the ${ }^{1} \pi \pi^{*}$ to the ${ }^{3} n \pi^{*}$ state followed by internal conversion to the ${ }^{3} \pi \pi^{*}$ state.

In contrast, a study by Yamazaki et al. for para-methoxy methylcinnamate ( $p-\mathrm{MMC})$, a close structural derivative of $p$-HMC, suggests that multistep intersystem crossing (ISC) from the ${ }^{1} \pi \pi^{*}$ state to the ${ }^{3} \pi \pi^{*}$ state could involve an intermediate ${ }^{3} n \pi^{*}$ state [25]. Similarly, a recent computational study by Kinoshita et al. suggests a major NRD route for $p$-HMC and 
$p$-MMC, based on MC, that supports the involvement of the aforementioned intermediate ${ }^{3} n \pi^{*}$ state [1]. Their rationale is based on the fact that $\mathrm{OH}$ and OMe are $\pi$ donors; the highest occupied molecular orbital $(\pi)$ is destabilised by these $\pi$-donors, reducing the $\pi$ - $\pi^{*}$ energy gap and consequently decreasing the energy of the $\pi \pi^{*}$ states while having minimal effect on the $n \pi^{*}$ states. This relative decrease in energy of the $\pi \pi^{*}$ states reverses the adiabatic ordering of the ${ }^{1} \pi \pi^{*}$ and ${ }^{1} \mathrm{n} \pi^{*}$ states, opening new major NRD routes. For $p$-HMC and $p$-MMC, Kinoshita et al. suggest the major NRD shown in Figure $2 \mathrm{~b}$.

There are four conformers of $p$-HMC, shown in Scheme 1. The $\mathrm{S}_{1} \leftarrow \mathrm{S}_{0}$ origin bands of these four conformers are located at 32,711.0, 32,715.9, 32,879.5, and $32,887.7 \mathrm{~cm}^{-1}$ for, respectively, (a), (b), (c) and (d) [26]. From the same study, Tan et al. used the linewidths from UV-UV depletion spectroscopy to propose lower lifetime limits of 1.5 and 2.5 ps for the s-cis and s-trans conformers, respectively. Since this work, Kinoshita et al. and Shimada et al. have carried out time-resolved pump-probe studies on $p$-HMC. In both of these studies, the ${ }^{1} \pi \pi^{*}$ lifetime was inferred to be $\leq 9$ ps $[27,28]$. The ${ }^{3} \pi \pi^{*}$ state has also been experimentally observed at $19,020 \mathrm{~cm}^{-1}$ and shown to have an average lifetime of $26 \pm 4$ ns [28]. However, the optically dark intermediate ${ }^{1} n \pi^{*}$ or ${ }^{3} n \pi^{*}$ state is lacking a direct observation, and therefore opens speculation for the major NRD route. This lack of observation may be attributed to weak $n \pi^{*} \leftarrow S_{0}$ absorption that is swamped by the strong $\mathrm{S}_{1}\left(\pi \pi^{*}\right) \leftarrow \mathrm{S}_{0}$ absorption in combination with a large ionisation energy to remove an electron from an $\mathrm{n} \pi^{*}$ state [28].
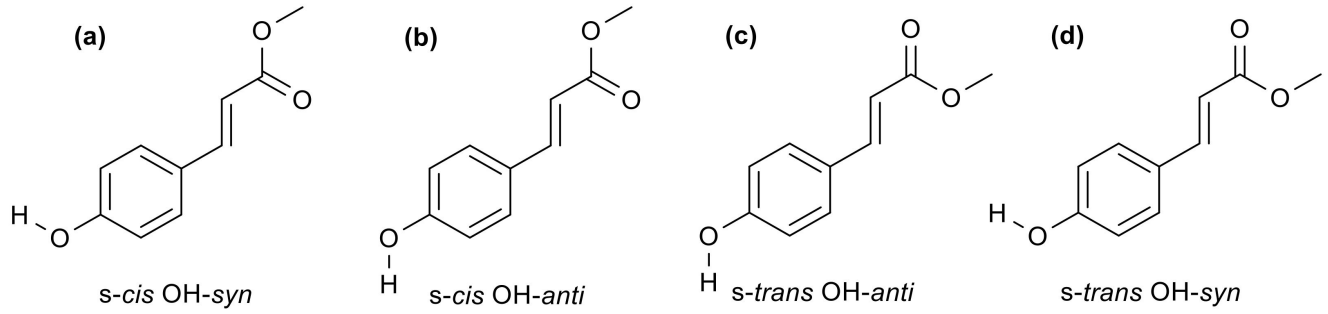

Scheme 1. Molecular structures for the four conformers of $p$-HMC: (a) s-cis OH-syn, (b) s-cis OH-anti, (c) s-trans $\mathrm{OH}-a n t i$, and (d) s-trans $\mathrm{OH}-\mathrm{syn}$.

In the present study, our aim is to: (a) precisely determine the gas-phase lifetime of the $S_{1}\left(\pi \pi^{*}\right)$ state in $p$-HMC; and (b) contribute towards determining the major NRD route from the $S_{1}\left(\pi \pi^{*}\right)$ state. To address (a), we use femtosecond (fs) to ps time-resolved ionyield (TR-IY) and time-resolved photoelectron spectroscopy (TR-PES). The approximate $200 \mathrm{~cm}^{-1}$ energy difference in the origin bands of the s-cis and s-trans conformers allows us to selectively excite and precisely measure the $S_{1}\left(\pi \pi^{*}\right)$ state lifetime of the s-cis $p$-HMC conformer. To address (b), time-dependent density functional theory (TDDFT) is used to calculate the possible relaxation pathways originating from the $S_{1}\left(\pi \pi^{*}\right)$ state in the s-cis conformer of $p$-HMC.

\section{Results and Discussion}

\subsection{Experimental}

Figure $3 b, c$ show TR-IY transients for the s-cis conformer of $p$-HMC including the associated time constants and errors for the forward dynamics (pump-probe time delay $(\Delta t)>0$ ), see Section 3.1. For these transients, the pump beam was centred at $308.5 \mathrm{~nm}$ $\left(32,415 \mathrm{~cm}^{-1}\right)$ to ensure s-cis (syn and anti) conformer-specific excitation of $p$-HMC to the $S_{1}\left(\pi \pi^{*}\right)$ state [26-28]. The spectral overlap of the pump beam with the $S_{1}\left(\pi \pi^{*}\right)$ state of relevant conformers can be seen in Figure 3a. For the TR-IY transient in Figure 3b, the probe beam was centred at $240 \mathrm{~nm}$ ( $p$-HMC's minimum absorption, Figure 1a) to minimise both the probe-only ion-signal and reverse dynamics $(\Delta t<0)$. The ionisation potential (IP) and the energy of the ${ }^{3} \pi \pi^{*}$ state are located around 65,020 and $19,020 \mathrm{~cm}^{-1}$, respectively $[27,28]$. As a result, a probe beam at $240 \mathrm{~nm}\left(41,667 \mathrm{~cm}^{-1}\right)$ ensures that ionisation is only optically 
accessible from the $S_{1}\left(\pi \pi^{*}\right)$ state (according to the proposed NRD paths shown in Figure 2), since the first two ionisation levels are of $\pi^{-1}$ character and there is insufficient energy to reach the $D_{2}\left(n^{-1}\right)$ ionisation state from any possible $n \pi^{*}$ states [28]. This is evidenced by the total kinetic energy release (TKER) spectra in Figure 4 (discussed below), derived from reconstructed photoelectron images (Figure 4 inset), which shows no real structural change upon varying $\Delta t$, apart from a fast $(<5 \mathrm{ps})$ decay. Knowing this, we can reasonably assume that the excited state lifetimes extracted from the TR-IY transient using a $240 \mathrm{~nm}$ probe in Figure $3 \mathrm{~b}$ are directly related to the $S_{1}\left(\pi \pi^{*}\right)$ state lifetime. For completeness, the probe beam in Figure $3 \mathrm{c}$ is centred at $200 \mathrm{~nm}\left(50,000 \mathrm{~cm}^{-1}\right)$ to enable ionisation from the $S_{1}\left(\pi \pi^{*}\right)$ state, any possible intermediate states, and the final ${ }^{3} \pi \pi^{*}$ excited state located $46,000 \mathrm{~cm}^{-1}$ below the IP. Since the ${ }^{3} \pi \pi^{*}$ state is known to have an average lifetime of $26 \pm 4 \mathrm{~ns}$, Figure $3 \mathrm{c}$ is expected to include an additional long-lived lifetime component (assuming it is formed within the time-window of our experiment, $900 \mathrm{ps}$ ) that would not be seen with the $240 \mathrm{~nm}$ probe due to the insufficient energy required to ionise from that state [28]. However, both transients exhibit the same dynamics, with three extracted time-constants $\tau_{1-3}$ using the procedure outlined in Section 3.1.
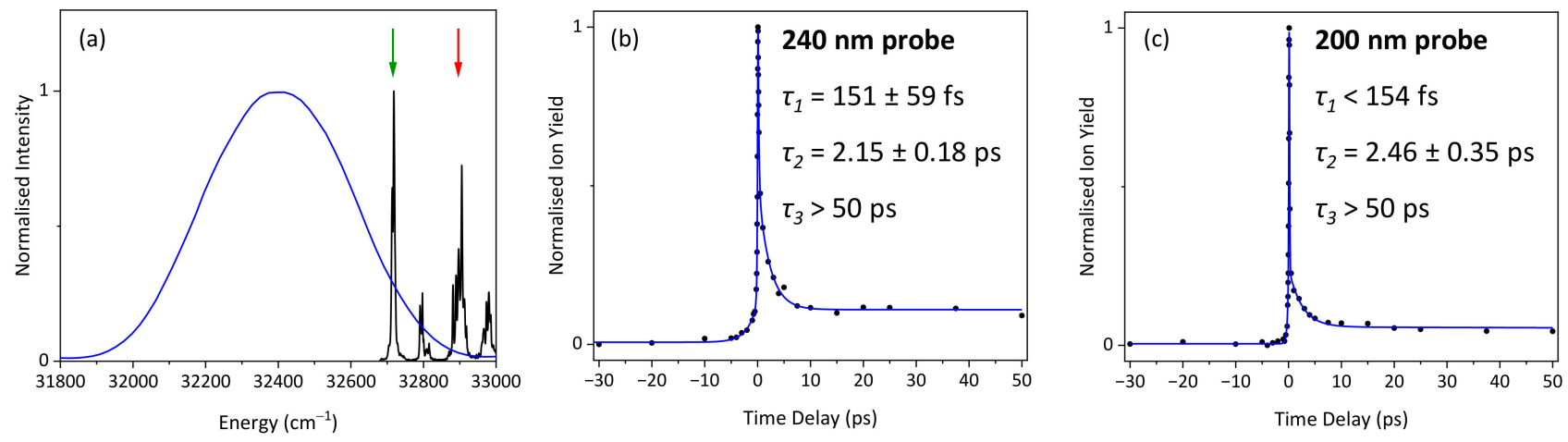

Figure 3. (a) Resonance enhanced multiphoton ionisation spectrum of $p$-HMC, relative to the $\mathrm{S}_{1}\left(\pi \pi^{*}\right)$ state, provided by Tan et al. [26]. The green arrow indicates the s-cis conformer and the red arrow indicates the s-trans conformer. Overlaid is the $308.5 \mathrm{~nm}\left(32,415 \mathrm{~cm}^{-1}\right)$ pump laser spectral profile which is used to selectively excite (predominantly) s-cis $p$-HMC to the $S_{1}\left(\pi \pi^{*}\right)$ state. (b) Time-resolved ion-yield (TR-IY) transient of $p$-HMC at $308.5 \mathrm{~nm}$ pump and $240 \mathrm{~nm}$ probe with a 3 bar helium backing pressure. (c) TR-IY transient of $p$-HMC at $308.5 \mathrm{~nm}$ pump and $200 \mathrm{~nm}$ probe with a $1.5 \mathrm{bar}$ helium backing pressure to reduce cluster formation (discussed infra). In $(\mathbf{b}, \mathbf{c})$, the polarisations of the pump and probe beams were parallel with respect to each other, and the negative dynamics were zeroed as the baseline fluctuated around zero due to poor background subtraction.

To start our discussion, we will address the first time constant, $\tau_{1}(\sim 150 \mathrm{fs})$. This lifetime is much shorter than the lower lifetime limit of 1.5 ps suggested by Tan et al. from the depletion spectra linewidth of the $S_{1}\left(\pi \pi^{*}\right)$ state in s-cis $p$-HMC, and is therefore likely not the lifetime of the $S_{1}\left(\pi \pi^{*}\right)$ state [26]. As mentioned supra, when using a $240 \mathrm{~nm}$ probe, the $S_{1}\left(\pi \pi^{*}\right)$ is the only state available for ionisation. Knowing this, we assign $\tau_{1}$ to initial evolution out of the Franck-Condon (FC) region, combined with any initial intramolecular vibrational redistribution, in keeping with previous studies [3,29-31].

The second time constant, $\tau_{2}$, from the TR-IY transients in Figure $3 b, c$ is $2.15 \pm 0.18$ ps and $2.46 \pm 0.35 \mathrm{ps}$, for a $240 \mathrm{~nm}$ and $200 \mathrm{~nm}$ probe, respectively. These lifetimes broadly agree with the lower limit of 1.5 ps suggested by Tan et al. and the $\leq 9$ ps suggested by Kinoshita et al. for the lifetime of the $S_{1}\left(\pi \pi^{*}\right)$ state in s-cis $p$-HMC [26,28]. Additionally, TR-PES (with a $240 \mathrm{~nm}$ probe) derived TKER spectra, shown in Figure 4, almost returns to baseline by 2 ps with no real structural change or shift in the maximum TKER (TKER $R_{\max }$ ). This implies that the ps decay is solely related to the decay of the initially populated excited

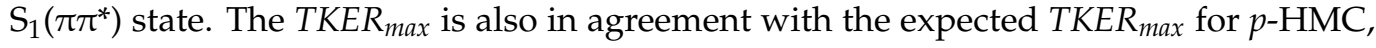


originating from excitation to the origin of s-cis p-HMC. Using Equation (1), the expected $T K E R_{\max }$ can be calculated:

$$
T K E R_{\max }=E_{O B}+E_{\text {probe }}-I P
$$

where $E_{O B}$ is the energy of the $S_{1}\left(\pi \pi^{*}\right)$ origin band for the s-cis conformers, $E_{\text {probe }}$ is the energy of the probe $\left(41,667 \mathrm{~cm}^{-1}\right)$, and $I P$ is the ionisation potential $\left(65,020 \mathrm{~cm}^{-1}\right)$. As a result, the expected TKER $\max$ is $9358 \mathrm{~cm}^{-1}$ and $9362.9 \mathrm{~cm}^{-1}$ for the two s-cis conformers, indicated by the black arrow in Figure 4 [26,27]. Encouragingly, this appears in excellent agreement with the observed TKER $R_{\max }$ from the TKER spectra shown in Figure 4. Considering these results and their agreement with previous results, $\tau_{2}$ is assigned to the lifetime of the $\mathrm{S}_{1}\left(\pi \pi^{*}\right)$ state of $p$-HMC in its s-cis configuration.

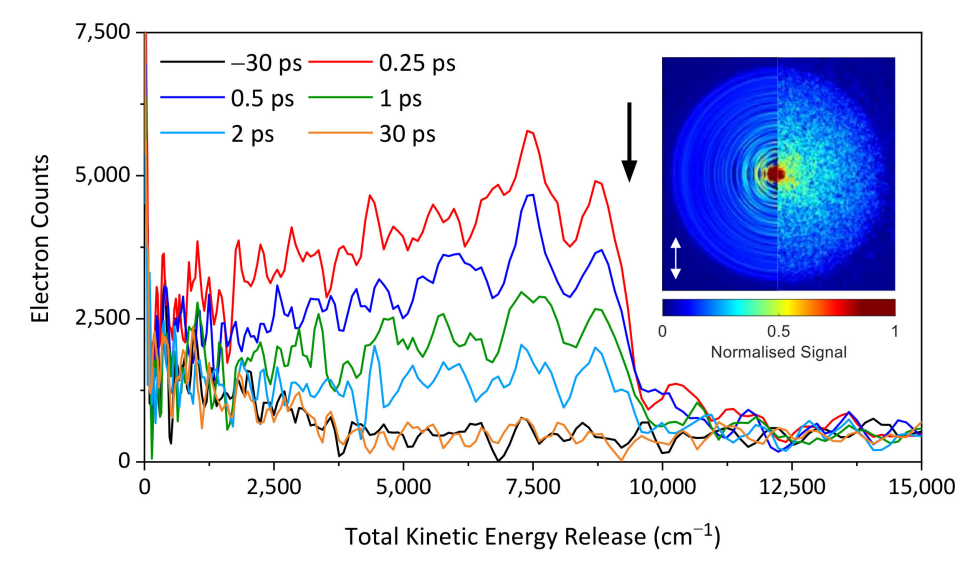

Figure 4. Total kinetic energy release (TKER) spectra of $p$-HMC obtained using the $308.5 \mathrm{~nm}$ pump and $240 \mathrm{~nm}$ probe with a 1.5 bar helium backing pressure. The following pump-probe time delays are used: $\Delta t=-30,0.25,0.5,1,2$ and $30 \mathrm{ps}$ and an expected $T K E R_{\max }$ of around $9360 \mathrm{~cm}^{-1}$ is indicated by the black arrow. Inset: right half presents the recorded image, while the left half presents the reconstructed slice through the centre of the original three-dimensional (3D) ion distribution at $\Delta t=0.25 \mathrm{ps}$ (the white arrow indicating the electric field polarisation of the laser pulses). The polarisations of the pump and probe beams were parallel with respect to each other.

Finally, the third time constant, $\tau_{3}$, is long-lived in both $240 \mathrm{~nm}$ probe and $200 \mathrm{~nm}$ probe transients and extends beyond the maximum time-window of our measurements, $900 \mathrm{ps}$ (ESI, Figures S1 and S2). When probing at $240 \mathrm{~nm}$, this result is surprising, since the only optically ionisable state is the $S_{1}\left(\pi \pi^{*}\right)$. As such, this merited further investigation. The relative amplitude of this feature is dependent on the helium backing pressure, which can be seen in Figure 5a for the TR-IY transients at 1.5 and 5 bar helium. An increase in the backing pressure increases collisional cooling, which leads to formation of water clusters, shown in Figure 5a inset. At 1.5 bar helium, most of the ion-signal is attributed to $p$-HMC, but at 5 bar helium, the data includes a large ion-signal that is assigned to $p$-HMC $+\mathrm{H}_{2} \mathrm{O}$ clusters. Furthermore, TKER spectra taken at 5 bar helium (Figure $5 b$ ), derived from reconstructed photoelectron images (Figure $5 \mathrm{~b}$ inset), are clearly different to those at 1.5 bar helium (Figure 4). The first notable difference is the increase in TKER $R_{\max }$ from approximately $9360 \mathrm{~cm}^{-1}$ to $>12,000 \mathrm{~cm}^{-1}$. The TKER $R_{\max }$ in Figure $5 \mathrm{~b}$ accords with the expected TKER $\max , 12,497 \mathrm{~cm}^{-1}$, when using an IP of $61,585 \mathrm{~cm}^{-1}$, determined by Smolarek et al., for a $p-\mathrm{HMC}+\mathrm{H}_{2} \mathrm{O}$ cluster (due to non-selective excitation, the expected $T K E R_{\max }$ is obtained using the pump energy and not the energy of the $S_{1}\left(\pi \pi^{*}\right)$ origin band for $p$-HMC $+\mathrm{H}_{2} \mathrm{O}$ ) [21]. From this, we can confirm the presence of water clusters at higher backing pressures. Additionally, it is clearly evident that the TKER spectra obtained at 5 bar helium do not tend to zero ( $c f$. at 1.5 bar helium); there is still population of the first excited state at $50 \mathrm{ps}$. This correlates strongly with the long-lived $\mathrm{S}_{1}\left(\pi \pi^{*}\right)$ state lifetime 
for the $p-\mathrm{HMC}+\mathrm{H}_{2} \mathrm{O}$ cluster, previously measured by ps pump-probe spectroscopy [27]. Considering the above, the marked baseline offset in the TR-IY transients (notably with a $240 \mathrm{~nm}$ probe) described by the long-lived component $\tau_{3}$, is attributed to the $S_{1}\left(\pi \pi^{*}\right)$ state in $p$ - $\mathrm{HMC}+\mathrm{H}_{2} \mathrm{O}$ clusters.
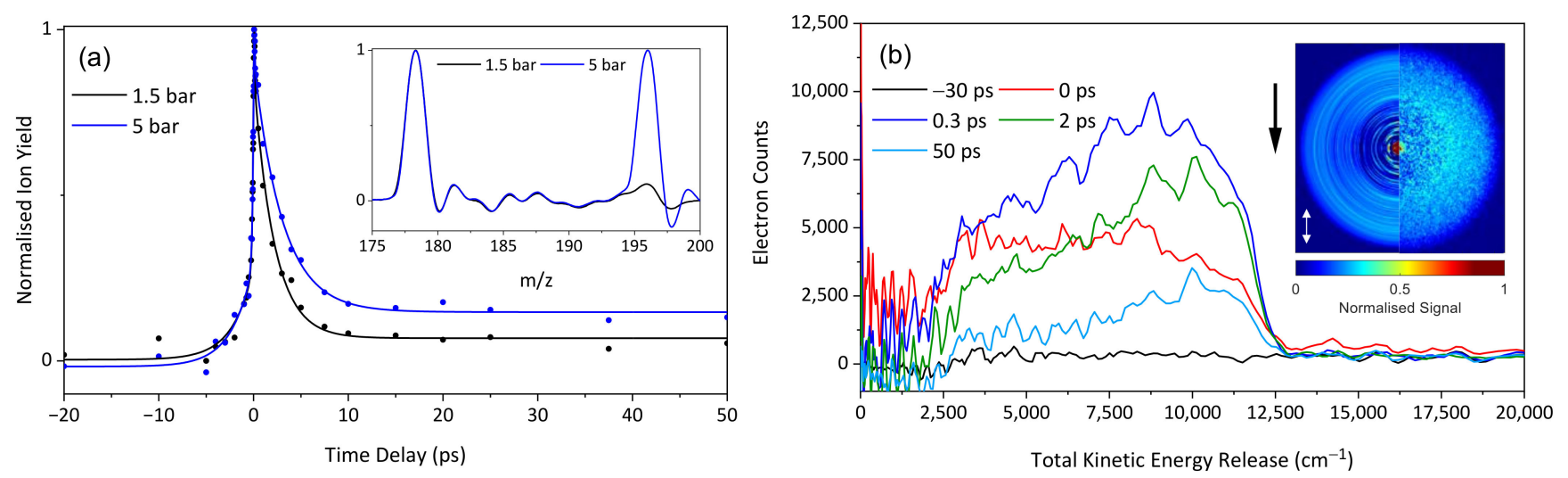

Figure 5. (a) TR-IY transient of $p$-HMC at $308.5 \mathrm{~nm}$ pump and $240 \mathrm{~nm}$ probe with a 1.5 and 5 bar helium backing pressure showing a larger baseline offset at 5 bar. Inset: time-of-flight mass spectrum, taken at 1.5 and 5 bar, showing the majority of ion-signal is $p$ - $\mathrm{HMC}^{+}(\mathrm{m} / \mathrm{z}=178)$ at 1.5 bar and at 5 bar, ion-signal corresponding to $\left[p-\mathrm{HMC}+\mathrm{H}_{2} \mathrm{O}\right]^{+}(\mathrm{m} / \mathrm{z}=196)$ additionally appears. (b) TKER spectra of $p$-HMC obtained using the $308.5 \mathrm{~nm}$ pump and $240 \mathrm{~nm}$ probe with a 5 bar helium backing pressure. The following pump-probe time delays are used: $\Delta t=-30,0,0.3,2$ and $50 \mathrm{ps}$ and an expected TKER max of $12,497 \mathrm{~cm}^{-1}$ is indicated by the black arrow. Inset: right half presents the recorded image, while the left half presents the reconstructed slice through the centre of the original 3D ion distribution at $\Delta t=0.3 \mathrm{ps}$ (the white arrow indicating the electric field polarisation of the laser pulses). For all data, the polarisations of the pump and probe beams were parallel with respect to each other.

We end this section with a caveat: When probing at $200 \mathrm{~nm}$, the mild baseline offset is expected since we can, potentially, ionise from both the ${ }^{1} n \pi^{*}$ and ${ }^{3} \pi \pi^{*}$ states. Consequently, we are unable to determine whether $\tau_{3}$ from the $200 \mathrm{~nm}$ probe TR-IY transient (see Figure $3 c$ ) is a result of cluster dynamics, ionisation from an $n \pi^{*}$ intermediate state, a ${ }^{3} \pi \pi^{*}$ state, or any of these combinations.

\subsection{Computational}

As the experiments were carried out on the s-cis conformers of $p$-HMC, we have carried out calculations using both $\mathrm{OH}-\mathrm{syn}$ and $\mathrm{OH}$-anti conformations (Scheme 1a,b), respectively); for brevity we will refer to these as syn and anti in what follows.

Initially the ground state geometries of the two conformers were optimised using density functional theory (DFT) and the excited state energies evaluated at both points. It was found that the anti conformation had the lower $\mathrm{S}_{0}$ energy at the minimum by $38 \mathrm{~cm}^{-1}$. As such, all energies reported here are given relative to this minimum, whichever conformation they are. We are interested in the NRD mechanism of this molecule, specifically whether the ${ }^{1} n \pi^{*}$ or ${ }^{3} n \pi^{*}$ state plays an intermediate role in that decay, and, as such, we note that, in both conformations, the ${ }^{1} \mathrm{n} \pi^{*}$ is the ninth lowest energy excited state (see data in ESI, Tables S1 and S2). There are five triplets below the bright $\mathrm{S}_{1} /{ }^{1} \pi \pi^{*}$ state, followed by a sixth triplet before two more singlets, $S_{2}$ being a less bright (by a factor of over 4 ) ${ }^{1} \pi \pi^{*}$ state and $S_{3}$ being the dark ${ }^{1} n \pi^{*}$ of interest (the non-bonding orbital contains the lone pair on the carbonyl oxygen, see Scheme 1). Above these two singlets is another triplet state, which is the limit of our interest. We note that $T_{5}$ in both conformations is of $n \pi^{*}$ character, whilst all other triplets have $\pi \pi^{*}$ character. It is thus clear that excitation will be predominantly to the $S_{1}$ state, that being by far the brightest in both cases. The vertical excitation energies are $39,993 \mathrm{~cm}^{-1}$ (anti) and $39,844 \mathrm{~cm}^{-1}$ (syn), meaning that, whilst the absolute values of the excitations are about $7000 \mathrm{~cm}^{-1}$ above the experimental values 
(which are adiabatic, so not directly comparable), they are close in energy with the correct ordering. In both cases, we also note that there is less than $3000 \mathrm{~cm}^{-1}$ between all three singlet excited states.

Given the two optimised, FC geometries, they were then used as starting points for optimisation on the excited states: minimum energy geometries on all ten excited states were sought (using restricted Kohn-Sham (RKS)), as were the CIs between all energy adjacent states of the same multiplicity (using RKS), and finally all crossings between energy adjacent states, regardless of multiplicity (using unrestricted Kohn-Sham (UKS), which does not distinguish states based on multiplicity in ORCA). We note that not all optimisations were successful, with some minima not being located in spite of restarted calculations at the most recently visited geometry. All data is included in Tables S1-S5 in the ESI (where the data is presented in energy order and labelled $S_{x}$ and $T_{y}$ for clarity, because the character of the states change at different geometries), which gives energies relative to the anti $\mathrm{S}_{0}$ minimum, but the main data to be discussed in what follows is shown in Figures 6 and 7. Here, Figure 6 contains information about geometries optimised from the syn conformer and Figure 7 contains information about points found starting with the anti conformer. We will focus our discussion on these selected points that best relate to the NRD of s-cis p-HMC. With these selected points, we have also tried to locate the transition states (TSs) between relevant pairs of conformers in order to assess any energy barriers between them. To note, there appears to be no energy barrier from the anti FC to the syn FC point. Therefore, initial excitation to the anti conformer could be followed by rotation of the $\mathrm{OH}$ group to the syn conformer, prior to any excited state crossings.

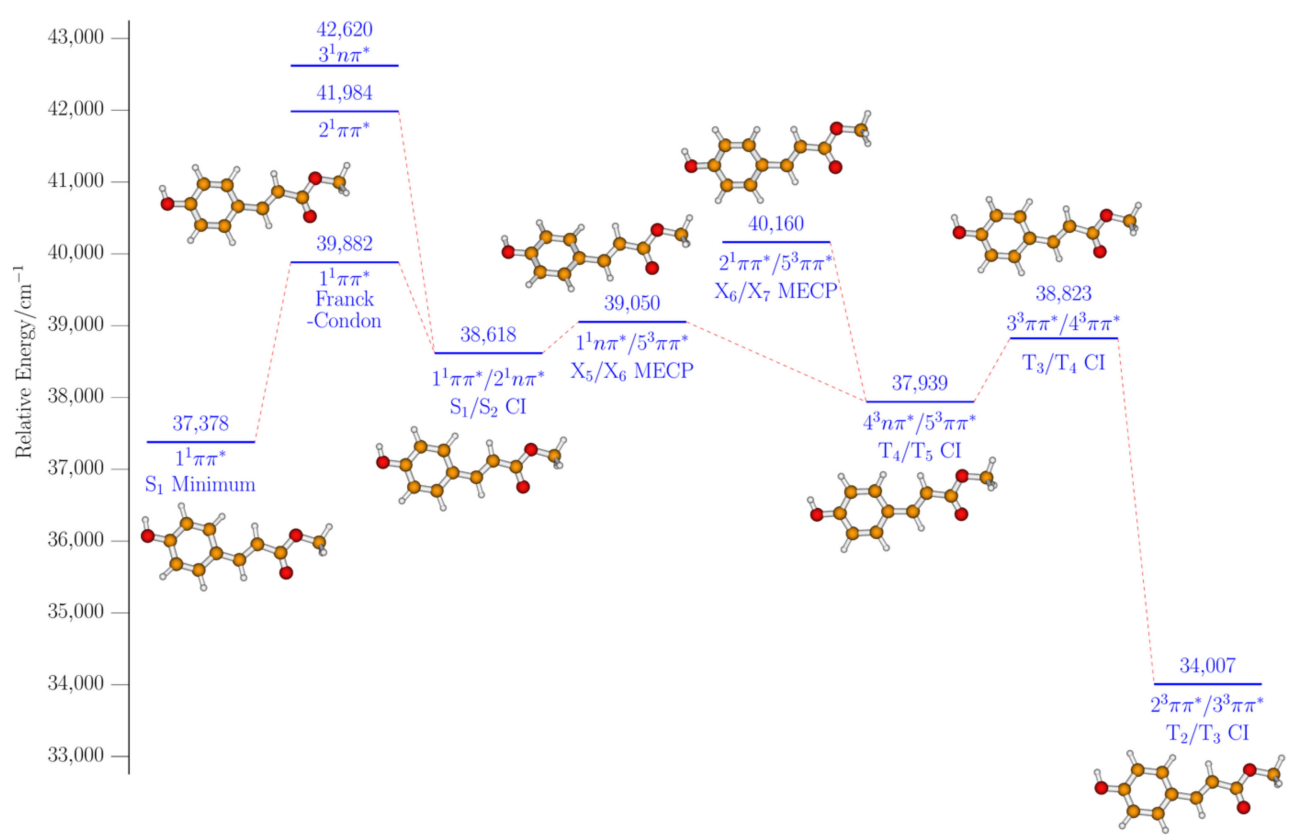

Figure 6. Energy landscape showing points relevant to the NRD of p-HMC. Geometries were optimised starting in the syn conformation. All energies are relative to the $\mathrm{S} 0$ state of the anti conformer Franck-Condon (FC) point. Energies, in $\mathrm{cm}-1$, are given above the blue lines; immediately below are the states in question; and below that are the labels of the optimised points. We have also included images of the molecular structures of the optimised points, above or below the lines for clarity. From left to right are the data for the S1 minimum; the FC point; the S1/S2 conical intersection (CI); the X5/X6 (S1/T5) minimum energy crossing point (MECP); the X6/X7 (S2/T5) MECP; the T4/T5 CI; the T3/T4 CI; and the T2/T3 CI. 


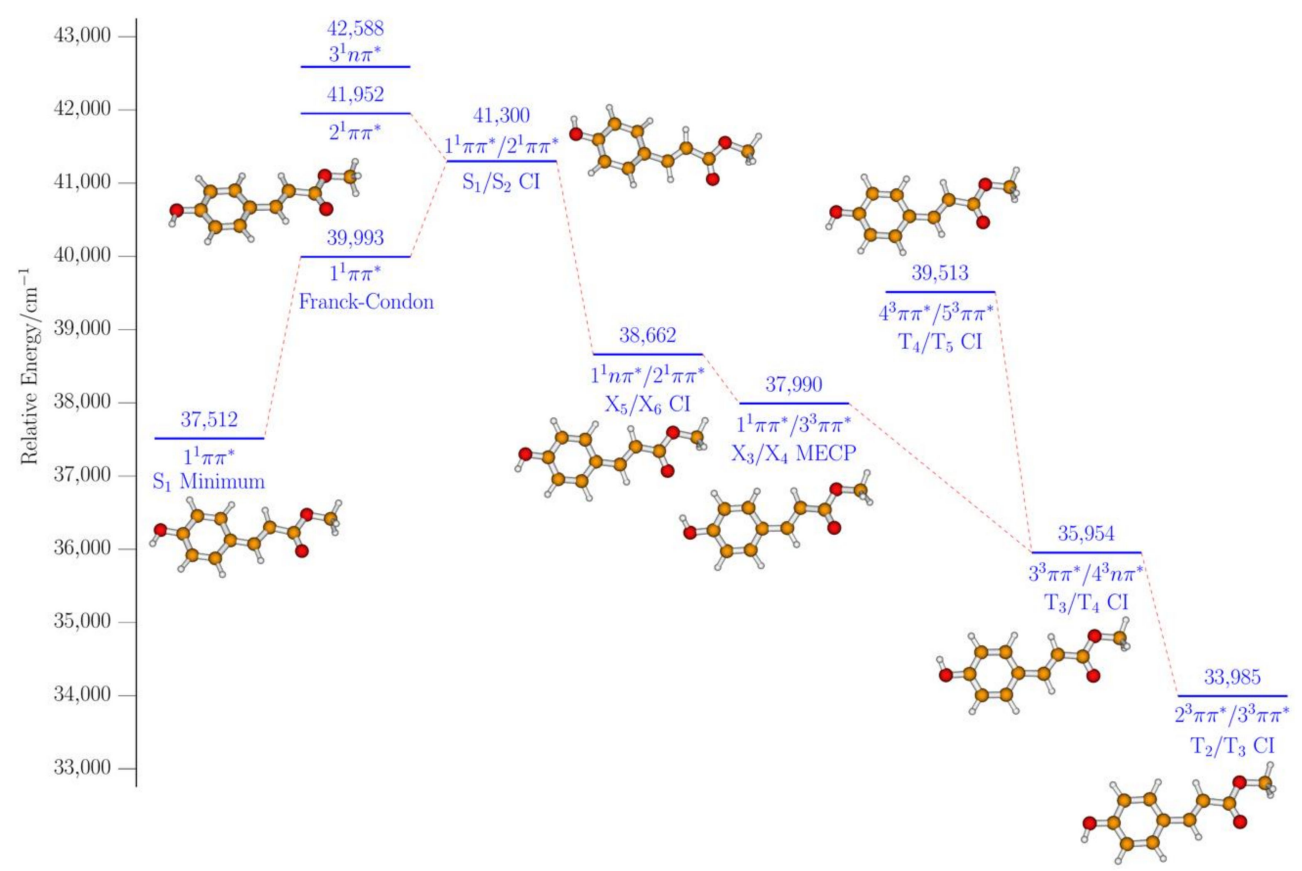

Figure 7. Energy landscape showing points relevant to the NRD of $p$-HMC. Geometries were optimised starting in the anti conformation. All energies are relative to the $\mathrm{S}_{0}$ state of the anti conformer FC point. Energies, in $\mathrm{cm}^{-1}$, are given above the blue lines; immediately below are the states in question; and below that are the labels of the optimised points. We have also included images of the molecular structures of the optimised points, above or below the lines for clarity. From left to right are the data for the $\mathrm{S}_{1}$ minimum; the FC point; the $\mathrm{S}_{1} / \mathrm{S}_{2} \mathrm{CI}$; the $\mathrm{X}_{5} / \mathrm{X}_{6}\left(\mathrm{~S}_{1} / \mathrm{S}_{2}\right) \mathrm{CI}$; the $\mathrm{X}_{3} / \mathrm{X}_{4}\left(\mathrm{~S}_{1} / \mathrm{T}_{3}\right) \mathrm{MECP}$; the $\mathrm{T}_{4} / \mathrm{T}_{5} \mathrm{CI}$; the $\mathrm{T}_{3} / \mathrm{T}_{4} \mathrm{CI}$; and the $\mathrm{T}_{2} / \mathrm{T}_{3} \mathrm{CI}$.

Looking at the deactivation of $p$-HMC by crossing to lower energy states, there are two proposed mechanisms, both of which involve ISC to the triplet manifold through which deactivation to $S_{0}$ occurs; the difference between the two is whether the first step is internal conversion to a ${ }^{1} \mathrm{n} \pi^{*}$ state before the ISC step or whether the excited molecule goes from ${ }^{1} \pi \pi^{*}$ to ${ }^{3} \pi \pi^{*}$ via ${ }^{3} n \pi^{*}$. To assess this, we first look at the ISC process to see what feasible paths there are by examining the spin-orbit couplings (SOCs) between the singlet and triplet states at the minimum energy crossing points (MECPs) found using UKS TDDFT. A total of ten singlet-triplet MECPs were found, four optimisations starting in the anti conformer and six in the syn. In what follows, we will denote the MECPs found by the initial conformer (anti or syn) and the crossed states, so anti-1 $1 \pi^{*} / 3^{3} \pi \pi^{*}$ is the crossing of $1^{1} \pi \pi^{*}$ and $3^{3} \pi \pi^{*}$ starting from the anti-FC point. The optimised anti-1 $1^{1} \pi \pi^{*} / 3^{3} \pi \pi^{*}$ geometry finished as a syn conformer, whilst all other nine systems stayed in their original anti or syn conformation. The anti-1 $1^{1} \pi \pi^{*} / 3^{3} \pi \pi^{*}$ MECP geometry is non-planar with carbonyl C (Scheme 1 ) having a dihedral angle of $165^{\circ}$ with the ring. The state energies at this MECP are similar to those found at $s y n-1^{1} \pi \pi^{*} / 3^{3} \pi \pi^{*}$ point, being only a few hundred $\mathrm{cm}^{-1}$ different in all cases. The syn $-1{ }^{1} \pi \pi^{*} / 3^{3} \pi \pi^{*}$ geometry has a dihedral angle of $-178.5^{\circ}$ between the carbonyl $\mathrm{C}$ and the ring, so the geometries of the MECPs are different, suggesting they lie on different seams of intersection.

Looking at the SOCs between the singlet and triplet states, shown in Table 1, the only couplings with a magnitude of greater than $1 \mathrm{~cm}^{-1}$ are at the anti-1 $\pi \pi^{*} / 3^{3} \pi \pi^{*}$ crossing $\left(1.27 \mathrm{~cm}^{-1}\right)$, the $s y n-2^{1} \pi \pi^{*} / 5^{3} \pi \pi^{*} \operatorname{MECP}\left(4.71 \mathrm{~cm}^{-1}\right)$ and the $\operatorname{syn}-1{ }^{1} \mathrm{n} \pi^{*} / 5^{3} \pi \pi^{*}$ geometry $\left(11.25 \mathrm{~cm}^{-1}\right)$. As already noted, the anti-1 $\pi \pi^{*} / 3^{3} \pi \pi^{*}$ geometry is actually synlike. We would expect the greatest rates of ISC to occur at these three crossing points, so let us consider the characters of the states there in more detail. At the anti-1 $\pi \pi^{*} / 3^{3} \pi \pi^{*}$ MECP, both intersecting states have $\pi \pi^{*}$ character. At the $s y n-1^{1} n \pi^{*} / 5^{3} \pi \pi^{*} \mathrm{MECP}$, the 
singlet is over $84 \% \mathrm{n} \pi^{*}$ with a little $\pi \pi^{*}$ mixed in, whilst at $\operatorname{syn}-2^{1} \pi \pi^{*} / 5^{3} \pi \pi^{*}$, the singlet is predominantly $\pi \pi^{*}$, but with over $20 \% \mathrm{n} \pi^{*}$ character included. From this analysis, we conclude that the largest SOCs at the MECPs are between singlets with significant $n \pi^{*}$ components in their character (the more the better) and $\pi \pi^{*}$ triplets. This is in accordance with El-Sayed's rule and therefore, unsurprising [32]. Pictures of the orbitals involved in the excitations, with largest components, which characterise the states that cross at the two geometries with largest SOCs are given in Figure S6 of the ESI. Even the anti-1 $1^{1} \pi \pi^{*} / 3^{3} \pi \pi^{*}$ MECP noted here has a SOC an order of magnitude lower than that at the $s y n-1^{1} n \pi^{*} / 5^{3} \pi \pi^{*}$ geometry. It thus appears that the rate of ISC will be greater going from a ${ }^{1} \mathrm{n} \pi^{*}$ state to a ${ }^{3} \pi \pi^{*}$ state in the triplet manifold, rather than from the ${ }^{1} \pi \pi^{*}$ state, suggesting that the NRD of $p$-HMC is mediated by the ${ }^{1} \mathrm{n} \pi^{*}$ state. In order for this to occur, there must be a route from the initially excited ${ }^{1} \pi \pi^{*}$ state to the ${ }^{1} \mathrm{n} \pi^{*}$, i.e., we need a CI and also a path to the crossing points from the FC point with a low barrier or none at all. We note that none of the other MECPs between singlet and triplet states, as listed in Table 1 , involved the ${ }^{3} \mathrm{n} \pi^{*}$ state, meaning that we have not located a path from the optically bright ${ }^{1} \pi \pi^{*}$ state to the ${ }^{3} n \pi^{*}$ state, which was the alternative NRD pathway proposed (Figure $2 b$ ). If such a crossing could be located, NRD by this route would have to compete with internal conversion to the ${ }^{1} \mathrm{n} \pi^{*}$ state, but we would expect this spin-allowed transition to occur more quickly than the spin-forbidden ISC to a ${ }^{3} n \pi^{*}$ state.

Table 1. Spin-orbit couplings between singlet and triplet states of $p$-HMC calculated at MECPs found by optimising geometries from both the anti and syn conformer FC points. Matrix elements for the $\mathrm{x}$, $\mathrm{y}$ and $\mathrm{z}$ components (all imaginary) given along with the magnitudes.

\begin{tabular}{cccccc}
\hline \multirow{2}{*}{$\begin{array}{c}\text { Initial } \\
\text { Geometry }\end{array}$} & MECP & \multicolumn{3}{c}{ Spin Orbit Coupling Values (cm $\left.\mathbf{c m}^{-1}\right)$} \\
\cline { 3 - 5 } Anti & $\mathbf{S} \rightarrow \mathbf{T}(\mathbf{x})$ & $\mathbf{S} \rightarrow \mathbf{T}(\mathbf{y})$ & $\mathbf{S} \rightarrow \mathbf{T}(\mathbf{z})$ & Magnitude \\
\hline & $1^{1} \pi \pi^{*} / 3^{3} \pi \pi^{*}\left(\mathrm{~S}_{1} / \mathrm{T}_{3}\right)$ & $0.66 \mathrm{i}$ & $0.52 \mathrm{i}$ & $-0.95 \mathrm{i}$ & 1.27 \\
& $1^{1} \pi \pi^{*} / 6^{3} \pi \pi^{*}\left(\mathrm{~S}_{1} / \mathrm{T}_{6}\right)$ & $0.01 \mathrm{i}$ & 0.00 & $-0.02 \mathrm{i}$ & 0.02 \\
& $2^{1} \pi \pi^{*} / 6^{3} \pi \pi^{*}\left(\mathrm{~S}_{2} / \mathrm{T}_{6}\right)$ & 0.00 & 0.00 & $0.01 \mathrm{i}$ & 0.01 \\
\multirow{3}{*}{ Syn } & $3^{1} \pi \pi^{*} / 7^{3} \pi \pi^{*}\left(\mathrm{~S}_{3} / \mathrm{T}_{7}\right)$ & 0.00 & 0.00 & $-0.01 \mathrm{i}$ & 0.01 \\
& $1^{1} \pi \pi^{*} / 3^{3} \pi \pi^{*}\left(\mathrm{~S}_{1} / \mathrm{T}_{3}\right)$ & $0.02 \mathrm{i}$ & $0.71 \mathrm{i}$ & $-0.06 \mathrm{i}$ & 0.71 \\
& $1^{1} \pi \pi^{*} / 4^{3} \pi \pi^{*}\left(\mathrm{~S}_{1} / \mathrm{T}_{4}\right)$ & $0.19 \mathrm{i}$ & $-0.53 \mathrm{i}$ & $-0.02 \mathrm{i}$ & 0.56 \\
& $1^{1} n \pi^{*} / 5^{3} \pi \pi^{*}\left(\mathrm{~S}_{1} / \mathrm{T}_{5}\right)$ & $4.29 \mathrm{i}$ & $10.28 \mathrm{i}$ & $1.58 \mathrm{i}$ & 11.25 \\
& $2^{1} \pi \pi^{*} / 5^{3} \pi \pi^{*}\left(\mathrm{~S}_{2} / \mathrm{T}_{5}\right)$ & $-0.59 \mathrm{i}$ & $4.57 \mathrm{i}$ & $0.99 \mathrm{i}$ & 4.71 \\
& $2^{1} \pi \pi^{*} / 6^{3} \pi \pi^{*}\left(\mathrm{~S}_{2} / \mathrm{T}_{6}\right)$ & $0.01 \mathrm{i}$ & 0.00 & 0.00 & 0.01 \\
& $3^{1} \pi \pi^{*} / 7^{3} \pi \pi^{*}\left(\mathrm{~S}_{3} / \mathrm{T}_{7}\right)$ & $-0.01 \mathrm{i}$ & 0.00 & 0.00 & 0.01 \\
\hline
\end{tabular}

A total of three CIs between the two lowest energy excited singlet states were found, two directly by searching for one starting at the FC geometries (anti-1 $1^{1} \pi \pi^{*} / 2^{1} \pi \pi^{*}$ and syn$1^{1} \pi \pi^{*} / 2^{1} n \pi^{*}$ ) and the third in the search for crossing using UKS TDDFT (anti-1 $1^{1} n \pi^{*} / 2^{1} \pi \pi^{*}$ ). Considering first the anti-1 $1^{1} \pi \pi^{*} / 2^{1} \pi \pi^{*}$ point, this has an energy directly between the energies of the $1^{1} \pi \pi^{*}$ and $2^{1} \pi \pi^{*}$ states at the anti-FC geometry, corresponding to $2800 \mathrm{~cm}^{-1}$ above the $1^{1} \pi \pi^{*}$ excitation point. The crossing would thus likely be energetically accessible in the experiment, however, we see that the character of the two states is ${ }^{1} \pi \pi^{*}$, i.e., it does not allow for crossing to the ${ }^{1} n \pi^{*}$ state. We also note that the final geometry has a syn conformation; the $\mathrm{OH}$ bond now points in the opposite direction to its original position. Next, we consider the CIs labelled syn-1 $1^{1} \pi \pi^{*} / 2^{1} n \pi^{*}$ and anti-1 $1^{1} n \pi^{*} / 2^{1} \pi \pi^{*}$, both of which have very similar energies (separated by about $50 \mathrm{~cm}^{-1}$ ) suggesting that the main difference between them is the orientation of the $\mathrm{OH}$ bond. The crossings are below the energies of the $1^{1} \pi \pi^{*}$ state at either FC point, so are definitely energetically accessible after excitation. With regard to the state characters, both crossings are between the ${ }^{1} n \pi^{*}$ state (greater than $85 \%$ components in both cases) and the bright ${ }^{1} \pi \pi^{*}$ state, so they represent an accessible route from the initially excited state and the ${ }^{1} \mathrm{n} \pi^{*}$ state which can, later on, give access to the triplet manifold. 
We have thus found a possible path from the bright $1^{1} \pi \pi^{*}$ state to the ${ }^{3} \pi \pi^{*}$ manifold and we also note that there are decreasing energy CIs between the triplet states which provide a path from $T_{5}$ to $T_{2}$, so there is a path towards the ground state. We have been unable to locate a crossing between the lowest two energy triplet states (except a very high energy crossing at a distorted geometry with the alkene-ester chain having migrated around the ring); however, that is not to say that one does not exist. With regard to the characters of the states at these crossing points: only the anti-3 $3^{1} n \pi^{*} / 4^{3} n \pi^{*}$ crossing involves the ${ }^{3} n \pi^{*}$ state. For the syn conformations, all states are ${ }^{3} \pi \pi^{*}$ at the triplet CIs, apart from the fourth triplet at the syn- $4^{3} n \pi^{*} / 5^{3} \pi \pi^{*} \mathrm{CI}$. As the crossing from the singlet manifold is ${ }^{1} \mathrm{n} \pi^{*}$ to ${ }^{3} \pi \pi^{*}$, it is not necessary for the decaying molecule to cross to the ${ }^{3} \mathrm{n} \pi^{*}$ at these CIs, the nuclear wavepacket may stay on the ${ }^{3} \pi \pi^{*}$ state as it makes its way down towards the lower states. Transfer of density to the ${ }^{3} n \pi^{*}$ state may compete with the decay through the ${ }^{3} \pi \pi^{*}$ states, slowing the NRD.

As a final set of calculations, we searched for barriers between relevant geometries. Of first interest was any barrier between the CIs found between the two lowest energy singlets (syn-1 $1^{1} \pi \pi^{*} / 2^{1} \mathrm{n} \pi^{*}$ and anti-1 ${ }^{1} \mathrm{n} \pi^{*} / 2^{1} \pi \pi^{*}$ ) and their corresponding FC geometries on the first singlet excited state potential energy surface. In both cases, the energies found were very close to that of the $1^{1} \pi \pi^{*}$ minimum $\left(\sim 2500 \mathrm{~cm}^{-1}\right.$ below the $1^{1} \pi \pi^{*}$ energy at the FC point), suggesting that no barrier is present. We note that there is also no barrier to the $1^{1} \pi \pi^{*}$ minimum energy geometry, thus there is likely to be competition between relaxation for the $1^{1} \pi \pi^{*}$ minimum and to the singlet CIs, potentially slowing the NRD. A barrier was also sought between the $s y n-1^{1} \pi \pi^{*} / 2^{1} n \pi^{*}$ geometry and the $\operatorname{syn}-1^{1} n \pi^{*} / 5^{3} \pi \pi^{*}$ point (i.e., the location of the largest SOC) and, again, none was found. This indicates that the path from the FC excitation geometry to the point of most likely ISC $\left(1^{1} n \pi^{*}\right.$ to $\left.5^{3} \pi \pi^{*}\right)$ is barrierless, the rate of NRD being determined by coupling between the states and the rate of competitive processes including relaxation to the $1^{1} \pi \pi^{*}$ minimum.

Bringing together the experimental and computational work, Figure 8 schematically illustrates the calculated major NRD route for s-cis $\mathrm{OH}-$ syn $p$-HMC along with the associated lifetimes, $\tau_{1}$ and $\tau_{2}$, determined by TR-IY and TR-PES. We focus specifically on the syn conformer since it shows a clearer path, with strong SOC, down through the inter-state crossings. Upon photoexcitation using a $308.5 \mathrm{~nm}$ pump, we propose that s-cis $p$-HMC evolves out of the FC region with no energy barrier in $\sim 150 \mathrm{fs}$. Subsequent to this, internal conversion to the ${ }^{1} \mathrm{n} \pi^{*}$ state occurs within $\sim 2.5 \mathrm{ps}$, and finally ISC to the ${ }^{3} \pi \pi^{*}$ state with no energy barrier.

\section{Materials and Methods}

\subsection{Experimental Setup}

The experimental setup has been described in detail elsewhere [23,33]. To briefly summarise, a Ti:Sapphire oscillator (Spectra-Physics Tsunami) and regenerative amplifier (Spectra-Physics Spitfire XP) are used to produce $\sim 40$ fs laser pulses at a rate of $1 \mathrm{kHz}$ and centred around $800 \mathrm{~nm}$. The output laser beam is $\sim 3 \mathrm{~W}$ and is subsequentially split into three equal $\sim 1 \mathrm{~W}$ parts. One $1 \mathrm{~W}$ part pumps an optical parametric amplifier (Light Conversion TOPAS-C) to produce the pump pulse, centred at $308.5 \mathrm{~nm}$ to ensure s-cis conformer selective excitation of $p$-HMC to the $S_{1}\left(\pi \pi^{*}\right)$ state. Another $1 \mathrm{~W}$ part either pumps a second TOPAS-C to generate the $240 \mathrm{~nm}$ probe or is successively frequency converted using a series of type I, type II and type I $\beta$-barium borate crystals to produce the $200 \mathrm{~nm}$ probe. For simplicity, the polarisations of the pump and probe are parallel with respect to each other, and the dynamics appear unaffected upon changing the probe to magic angle $\left(54.7^{\circ}\right)$ with respect to the pump-indicating the absence of rotational artifacts (ESI, Figure S3).

Both beams intersect a molecular beam produced by seeding $p$-HMC $(99.9 \%$ purity by HPLC, Apollo Scientific) heated to $170{ }^{\circ} \mathrm{C}$ into $1.5,3$ and 5 bar helium. This gas mixture is expanded into vacuum via an Even-Lavie pulsed solenoid valve and is subsequentially passed through a $2 \mathrm{~mm}$ diameter skimmer [34,35]. 


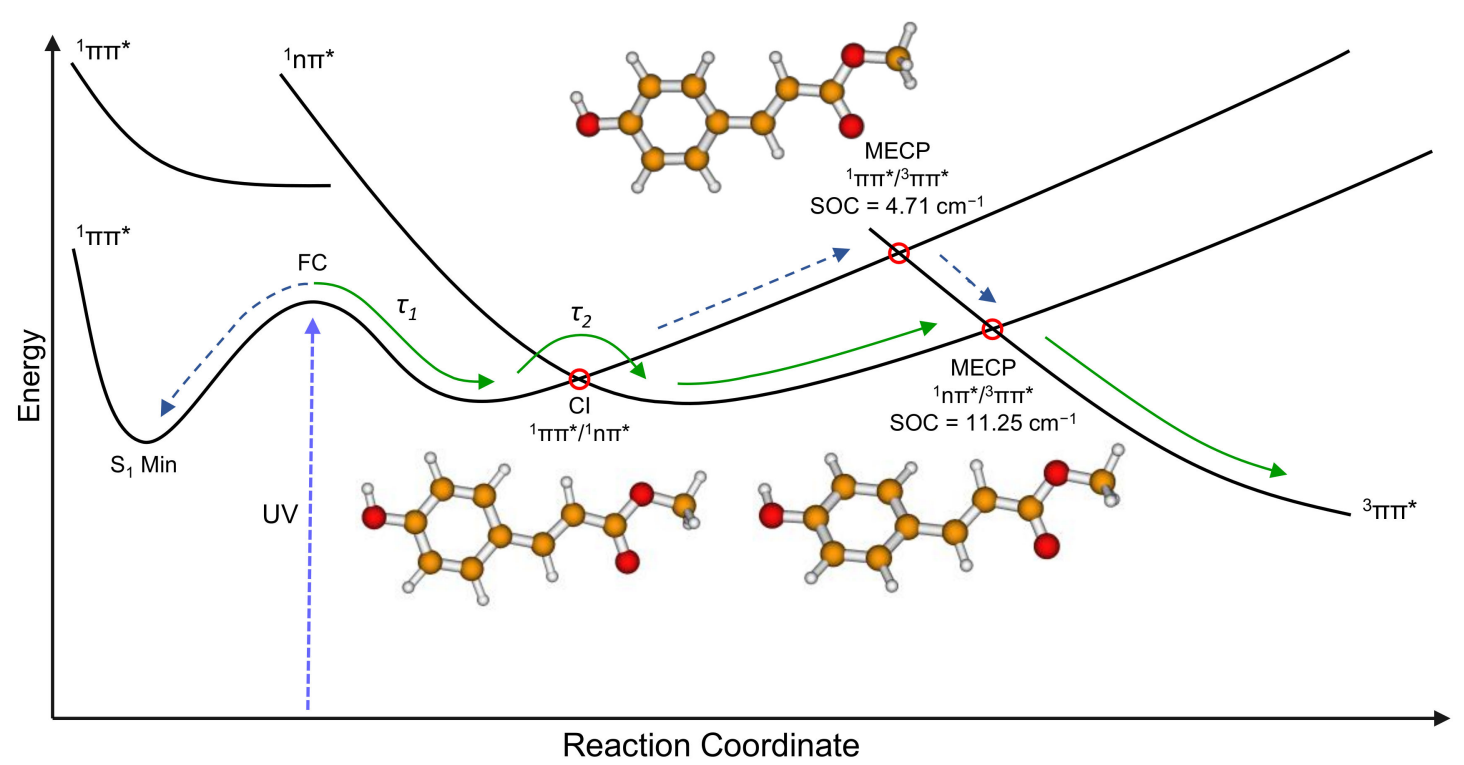

Figure 8. Potential energy surface schematic for s-cis OH-syn p-HMC, based on the energy landscape diagram in Figure 6. At the point of excitation, the vertical ordering of states is the first $1 \pi \pi^{*}$ state, the second $1 \pi \pi^{*}$ (not of dynamical interest) and the $1 \mathrm{n} \pi^{*}$ state. The green arrows represent the major NRD route after photoexcitation to the first $1 \pi \pi^{*}$ state, while the dark blue dotted arrows represent alternative or competing excited state processes. The processes identified experimentally are labelled $\tau 1$ and $\tau 2$ and relate to the lifetimes measured in Section 2.1. CI and MECP structures are shown along with the relevant spin-orbit coupling (SOC) values.

For TR-IY, the photoions are accelerated, via an electric field, towards a detector which consists of a $25 \mathrm{~mm}$ active area MCP with a metal anode detector (Del Mar Photonics MCP-MA25/2). The signal output is measured by a digital oscilloscope (LeCroy LT372 Waverunner) and the ion-signal relating to the parent ion $\left(p-\mathrm{HMC}^{+}\right)$is monitored as a function of $\Delta t$; this creates the TR-IY transients.

A velocity map ion imaging setup, based off a design by Eppink and Parker, is used for TR-PES [36]. The photoelectrons are accelerated and focused onto a position-sensitive temporally-gated detector, consisting of two MCPs coupled to a phosphor screen (Photek VID-240) and imaged by a CCD camera (Basler A-312f). In this configuration, electrons with the same initial velocity are mapped onto the same point on the detector. The twodimensional images generated are used to reconstruct the original three-dimensional Newton sphere using a polar onion peeling algorithm, from which the desired one-dimensional photoelectron spectrum is derived [37]. Xenon is used to calibrate the detector by which the obtained photoelectron spectrum is scaled to coincide with the energy of its well-known ionisation states [38].

Dynamical information from the TR-IY transients $(S(\Delta t)$ vs. $\Delta t)$ was extracted via a non-linear curve fitting algorithm consisting of a sum of exponential decay functions convoluted with the instrument response function (IRF):

$$
S(\Delta t)=S_{0}+G(\Delta t) *\left[\sum_{i} A_{i} e^{\left(-\frac{\Delta t-t_{0}}{\tau_{i}}\right)} H\left(\Delta t-t_{0}\right)\right]
$$

Here, $S_{0}$ is the signal baseline, $G(\Delta t)$ is the Gaussian function to account for the IRF, $A_{i}$ is the amplitude of the $i$-decay, $t_{0}$ is time zero, $\tau_{i}$ is the lifetime of the $i$-decay and $H\left(\Delta t-t_{0}\right)$ is a step function that is used to define time zero as the starting point of all observed dynamics; such that:

$$
H\left(\Delta t-t_{0}\right)= \begin{cases}0 & \text { if }\left(\Delta t-t_{0}\right)<0 \\ 1 & \text { if }\left(\Delta t-t_{0}\right) \geq 0\end{cases}
$$


The instrument response (IR) is estimated from the cross-correlation of the $308.5 \mathrm{~nm}$ pump and 240/200 nm probe with ammonia (ESI, Figures S4 and S5). From this, the IR is determined to be $117 \pm 3 \mathrm{fs}$ and $154 \pm 6 \mathrm{fs}$ with the $240 \mathrm{~nm}$ and $200 \mathrm{~nm}$ probe, respectively.

\subsection{Computational Details}

To further investigate the NRD of $p$-HMC, we have performed a series of electronic structure calculations to explore the ground- and excited-state potential energy surfaces of the molecule. To do so, we have used DFT for the ground state and TDDFT for the excited states, as implemented in the ORCA quantum chemistry package (version 5.0.1) [39-41], specifically using the $\omega \mathrm{B} 97 \mathrm{X}$ functional, both in RKS and UKS forms allied with the 6$311 \mathrm{G}(\mathrm{d}, \mathrm{p})$ basis set $[42,43]$. The molecular geometry has been optimised using RKS DFT and TDDFT in order to locate energy minima on the electronic ground and excited states (both singlet and triplet); at such points, frequency calculations were carried out to ensure that the geometries were indeed minima of the potential energy surfaces, rather than saddle points. Additionally, MECPs between excited states were located, these being CIs in the case of crossing between states of the same multiplicity (found using RKS TDDFT). Once these minima and MECPs had been found, further calculations were performed to locate TSs between selected geometries, on the states of interest, where such barriers exist. The initial guess for the TS structure was found by taking the central geometry in a linear interpolation between the two selected geometries (using their respective Zmatrices). Upon convergence of the TS structures using the energies of the state of interest, frequency calculations were performed on that state to ensure that all frequencies were real except for one; the single imaginary frequency indicates that the structure is indeed a TS. All such optimised geometries were indeed found to be TSs using this condition. At points of crossing between singlet and triplet states, SOCs were calculated to assess the probability of ISC at such geometries. These points were located using UKS TDDFT, but were then checked using RKS TDDFT, where state energies were found to be the same as when using UKS; the SOCs of these RKS states found with quasi-degenerate perturbation theory [44]. Tight convergence criteria were used both for the geometry optimisations (convergence criteria were: $10^{-6}$ Eh change in energy; $10^{-4} \mathrm{Eh} / \mathrm{Bohr}$ maximum absolute gradient component; $3 \times 10^{-5} \mathrm{Eh} /$ Bohr root mean square (RMS) of gradient components; $10^{-3}$ Bohr maximum coordinate displacement and $6 \times 10^{-4}$ Bohr RMS displacement) and the underlying electronic structure calculations (convergence criteria were: $10^{-8} \mathrm{Eh}$ energy change; $10^{-5}$ Eh change in the one-electron component of the energy; $10^{-5}$ orbital gradient, $10^{-5}$ orbital rotation angle; and $5 \times 10^{-7}$ error in the direct inversion of the iterative subspace).

\section{Conclusions}

We have investigated the gas-phase excited state dynamics of s-cis para-hydroxy methylcinnamate with both experiment and computation. Using pump-probe femtosecond time-resolved ion-yield and time-resolved photoelectron spectroscopies, the gas-phase $\mathrm{S}_{1}\left(\pi \pi^{*}\right)$ excited state lifetime was determined. Time-resolved ion-yield transients produced $\mathrm{S}_{1}\left(\pi \pi^{*}\right)$ lifetimes of $2.15 \pm 0.18 \mathrm{ps}$ and $2.46 \pm 0.35 \mathrm{ps}$ when probing at $240 \mathrm{~nm}$ and $200 \mathrm{~nm}$, respectively. These lifetimes sensibly compare with time-resolved photoelectron spectroscopy measurements. Furthermore, the possible relaxation pathways were calculated by time-dependent density functional theory. We found the major non-radiative route, following photoexcitation to the $S_{1}\left(\pi \pi^{*}\right)$ state, is mostly likely intersystem crossing to the ${ }^{3} \pi \pi^{*}$ state, mediated by an optically dark ${ }^{1} \mathrm{n} \pi^{*}$ state. Overall, the excited state lifetime and major non-radiative decay route from the first excited state in s-cis para-hydroxy methylcinnamate correlates strongly with structurally similar molecules, such as para-coumaric acid and methylcinnamate. This conclusion brings us closer to developing a greater understanding for the photodynamical behaviour of cinnamates and coumaric acids, to ultimately tailor similar molecules for applications including sunscreens and molecular heaters. 
Supplementary Materials: The following are available online. Figures S1 and S2: TR-IY transients up to 900 ps, Figure S3: TR-IY transient at magic angle, Figures S4 and S5: cross-correlation, Figure S6: molecular orbitals, Tables S1-S5: computational data.

Author Contributions: Conceptualization, J.D. and V.G.S.; formal analysis, J.D.; investigation, J.D., G.W.R., J.M.W. and T.T.A.; supervision, S.H. and V.G.S.; visualization, J.D. and G.W.R.; writingoriginal draft, J.D. and G.W.R.; writing-review and editing, J.D., G.W.R., J.M.W., T.T.A., S.H. and V.G.S. All authors have read and agreed to the published version of the manuscript.

Funding: The research conducted by G.W.R. and S.H. was funded by the Engineering and Physical Sciences Research Council grant EP/S028986/1.

Data Availability Statement: The data presented in this study is available in the article or supplementary material.

Acknowledgments: The authors would like to thank W.J. Buma (University of Amsterdam) for providing the resonance enhanced multiphoton ionisation spectrum of para-hydroxy methylcinnamate. G.W.R. and S.H. thank the Scientific Computing Research Technology Platform at the University of Warwick for providing computing facilities. V.G.S. thanks the Royal Society for a Royal Society Industry Fellowship.

Conflicts of Interest: The authors declare no conflict of interest.

\section{References}

1. Kinoshita, S.N.; Harabuchi, Y.; Inokuchi, Y.; Maeda, S.; Ehara, M.; Yamazaki, K.; Ebata, T. Substitution Effect on the Nonradiative Decay and Trans Cis Photoisomerization Route: A Guideline to Develop Efficient Cinnamate-Based Sunscreens. Phys. Chem. Chem. Phys. 2021, 23, 834-845. [CrossRef] [PubMed]

2. Promkatkaew, M.; Suramitr, S.; Karpkird, T.; Wanichwecharungruang, S.; Ehara, M.; Hannongbua, S. Photophysical Properties and Photochemistry of Substituted Cinnamates and Cinnamic Acids for UVB Blocking: Effect of Hydroxy, Nitro, and Fluoro Substitutions at Ortho, Meta, and Para Positions. Photochem. Photobiol. Sci. 2014, 13, 583-594. [CrossRef] [PubMed]

3. Peperstraete, Y.; Staniforth, M.; Baker, L.A.; Rodrigues, N.D.N.; Cole-Filipiak, N.C.; Quan, W.D.; Stavros, V.G. Bottom-up Excited State Dynamics of Two Cinnamate-Based Sunscreen Filter Molecules. Phys. Chem. Chem. Phys. 2016, 18, 28140-28149. [CrossRef] [PubMed]

4. Karpkird, T.M.; Wanichweacharungruang, S.; Albinsson, B. Photophysical Characterization of Cinnamates. Photochem. Photobiol. Sci. 2009, 8, 1455-1460. [CrossRef] [PubMed]

5. Iida, Y.; Kinoshita, S.N.; Kenjo, S.; Muramatsu, S.; Inokuchi, Y.; Zhu, C.; Ebata, T. Electronic States and Nonradiative Decay of Cold Gas-Phase Cinnamic Acid Derivatives Studied by Laser Spectroscopy with a Laser-Ablation Technique. J. Phys. Chem. A 2020, 124, 5580-5589. [CrossRef] [PubMed]

6. Meyer, T.E. Isolation and Characterization of Soluble Cytochromes, Ferredoxins and Other Chromophoric Proteins from the Halophilic Phototrophic Bacterium Ectothiorhodospira Halophila. Biochim. Biophys. Acta 1985, 806, 175-183. [CrossRef]

7. Meyer, T.E.; Fitch, J.C.; Bartsch, R.G.; Tollin, G.; Cusanovich, M.A. Soluble Cytochromes and a Photoactive Yellow Protein Isolated from the Moderately Halophilic Purple Phototrophic Bacterium, Rhodospirillum Salexigens. Biochim. Biophys. Acta 1990, 1016, 364-370. [CrossRef]

8. Meyer, T.E.; Yakali, E.; Cusanovich, M.A.; Tollin, G. Properties of a Water-Soluble, Yellow Protein Isolated from a Halophilic Phototrophic Bacterium That Has Photochemical Activity Analogous to Sensory Rhodopsin. Biochemistry 1987, 26, 418-423. [CrossRef] [PubMed]

9. Imamoto, Y.; Kataoka, M. Structure and Photoreaction of Photoactive Yellow Protein, a Structural Prototype of the PAS Domain Superfamily. Photochem. Photobiol. 2007, 83, 40-49. [CrossRef]

10. Carroll, E.C.; Hospes, M.; Valladares, C.; Hellingwerf, K.J.; Larsen, D.S. Is the Photoactive Yellow Protein a UV-B/Blue Light Photoreceptor? Photochem. Photobiol. Sci. 2011, 10, 464-468. [CrossRef] [PubMed]

11. Schlichting, I.; Berendzen, J. Out of the Blue: The Photocycle of the Photoactive Yellow Protein. Structure 1997, 5, 735-739. [CrossRef]

12. Sprenger, W.W.; Hoff, W.D.; Armitage, J.P.; Hellingwerf, K.J. The Eubacterium Ectothiorhodospira Halophila Is Negatively Phototactic, with a Wavelength Dependence That Fits the Absorption Spectrum of the Photoactive Yellow Protein. J. Bacteriol. 1993, 175, 3096-3104. [CrossRef] [PubMed]

13. Burnett, M.E.; Hu, J.Y.; Wang, S.Q. Sunscreens: Obtaining Adequate Photoprotection. Dermatol. Ther. 2012, 25, 244-251. [CrossRef] [PubMed]

14. Mancuso, J.B.; Maruthi, R.; Wang, S.Q.; Lim, H.W. Sunscreens: An Update. Am. J. Clin. Dermatol. 2017, 18, 643-650. [CrossRef] [PubMed]

15. Palm, M.D.; O'Donoghue, M.N. Update on Photoprotection. Dermatol. Ther. 2007, 20, 360-376. [CrossRef] [PubMed] 
16. De Groot, M.; Gromov, E.V.; Köppel, H.; Buma, W.J. High-Resolution Spectroscopy of Methyl 4-Hydroxycinnamate and Its Hydrogen-Bonded Water Complex. J. Phys. Chem. B 2008, 112, 4427-4434. [CrossRef] [PubMed]

17. Roberts, G.M.; Stavros, V.G. The Role of $\Pi \sigma^{*}$ States in the Photochemistry of Heteroaromatic Biomolecules and Their Subunits: Insights from Gas-Phase Femtosecond Spectroscopy. Chem. Sci. 2014, 5, 1698-1722. [CrossRef]

18. Sobolewski, A.L.; Domcke, W.; Dedonder-Lardeux, C.; Jouvet, C. Excited-State Hydrogen Detachment and Hydrogen Transfer Driven by Repulsive $1 \pi \sigma^{*}$ States: A New Paradigm for Nonradiative Decay in Aromatic Biomolecules. Phys. Chem. Chem. Phys. 2002, 4, 1093-1100. [CrossRef]

19. Ratzer, C.; Küpper, J.; Spangenberg, D.; Schmitt, M. The Structure of Phenol in the S1-State Determined by High Resolution UV-Spectroscopy. Chem. Phys. 2002, 283, 153-169. [CrossRef]

20. Dixon, R.N.; Oliver, T.A.A.; Ashfold, M.N.R. Tunnelling under a Conical Intersection: Application to the Product Vibrational State Distributions in the UV Photodissociation of Phenols. J. Chem. Phys. 2011, 134, 194303. [CrossRef] [PubMed]

21. Smolarek, S.; Vdovin, A.; Tan, E.M.M.; De Groot, M.; Buma, W.J. Spectroscopy and Dynamics of Methyl-4-Hydroxycinnamate: The Influence of Isotopic Substitution and Water Complexation. Phys. Chem. Chem. Phys. 2011, 13, 4393-4399. [CrossRef] [PubMed]

22. Smolarek, S.; Vdovin, A.; Perrier, D.L.; Smit, J.P.; Drabbels, M.; Buma, W.J. High-Resolution Excitation and Absorption Spectroscopy of Gas-Phase p-Coumaric Acid: Unveiling an Elusive Chromophore. J. Am. Chem. Soc. 2010, 132, 6315-6317. [CrossRef] [PubMed]

23. Krokidi, K.M.; Turner, M.A.P.; Pearcy, P.A.J.; Stavros, V.G. A Systematic Approach to Methyl Cinnamate Photodynamics. Mol. Phys. 2021, 119, e1811910. [CrossRef]

24. Kinoshita, S.N.; Inokuchi, Y.; Onitsuka, Y.; Kohguchi, H.; Akai, N.; Shiraogawa, T.; Ehara, M.; Yamazaki, K.; Harabuchi, Y.; Maeda, S.; et al. The Direct Observation of the Doorway $1 \mathrm{n} \pi *$ State of Methylcinnamate and Hydrogen-Bonding Effects on the Photochemistry of Cinnamate-Based Sunscreens. Phys. Chem. Chem. Phys. 2019, 21, 19755-19763. [CrossRef]

25. Yamazaki, K.; Miyazaki, Y.; Harabuchi, Y.; Taketsugu, T.; Maeda, S.; Inokuchi, Y.; Kinoshita, S.N.; Sumida, M.; Onitsuka, Y.; Kohguchi, H.; et al. Multistep Intersystem Crossing Pathways in Cinnamate-Based UV-B Sunscreens. J. Phys. Chem. Lett. 2016, 7, 4001-4007. [CrossRef]

26. Tan, E.M.M.; Amirjalayer, S.; Bakker, B.H.; Buma, W.J. Excited State Dynamics of Photoactive Yellow Protein Chromophores Elucidated by High-Resolution Spectroscopy and Ab Initio Calculations. Faraday Discuss. 2013, 163, 321-340. [CrossRef]

27. Shimada, D.; Kusaka, R.; Inokuchi, Y.; Ehara, M.; Ebata, T. Nonradiative Decay Dynamics of Methyl-4-Hydroxycinnamate and Its Hydrated Complex Revealed by Picosecond Pump-Probe Spectroscopy. Phys. Chem. Chem. Phys. 2012, 14, 8999-9005. [CrossRef]

28. Kinoshita, S.N.; Miyazaki, Y.; Sumida, M.; Onitsuka, Y.; Kohguchi, H.; Inokuchi, Y.; Akai, N.; Shiraogawa, T.; Ehara, M.; Yamazaki, K.; et al. Different Photoisomerization Routes Found in the Structural Isomers of Hydroxy Methylcinnamate. Phys. Chem. Chem. Phys. 2018, 20, 17583-17598. [CrossRef]

29. Kotsina, N.; Candelaresi, M.; Saalbach, L.; Zawadzki, M.M.; Crane, S.W.; Sparling, C.; Townsend, D. Short-Wavelength Probes in Time-Resolved Photoelectron Spectroscopy: An Extended View of the Excited State Dynamics in Acetylacetone. Phys. Chem. Chem. Phys. 2020, 22, 4647-4658. [CrossRef] [PubMed]

30. De Camillis, S.; Miles, J.; Alexander, G.; Ghafur, O.; Williams, I.D.; Townsend, D.; Greenwood, J.B. Ultrafast Non-Radiative Decay of Gas-Phase Nucleosides. Phys. Chem. Chem. Phys. 2015, 17, 23643-23650. [CrossRef]

31. Liu, Y.-Z.; Qin, C.-C.; Zhang, S.; Wang, Y.-M.; Zhang, B. Ultrafast Dynamics of the First Excited State of Chlorobenzene. Acta Phys. Chim. Sin. 2011, 27, 965-970.

32. El-Sayed, M.A. Spin-Orbit Coupling and the Radiationless Processes in Nitrogen Heterocyclics. J. Chem. Phys. 1963, 38, 2834-2838. [CrossRef]

33. Iqbal, A.; Pegg, L.J.; Stavros, V.G. Direct versus Indirect H Atom Elimination from Photoexcited Phenol Molecules. J. Phys. Chem. A 2008, 112, 9531-9534. [CrossRef] [PubMed]

34. Even, U. The Even-Lavie Valve as a Source for High Intensity Supersonic Beam. EPJ Tech. Instrum. 2015, 2, 1-22. [CrossRef]

35. Even, U.; Jortner, J.; Noy, D.; Lavie, N.; Cossart-Magos, C. Cooling of Large Molecules below 1 K and He Clusters Formation. J. Chem. Phys. 2000, 112, 8068-8071. [CrossRef]

36. Eppink, A.T.J.B.; Parker, D.H. Velocity Map Imaging of Ions and Electrons Using Electrostatic Lenses: Application in Photoelectron and Photofragment Ion Imaging of Molecular Oxygen. Rev. Sci. Instrum. 1997, 68, 3477-3484. [CrossRef]

37. Roberts, G.M.; Nixon, J.L.; Lecointre, J.; Wrede, E.; Verlet, J.R.R. Toward Real-Time Charged-Particle Image Reconstruction Using Polar Onion-Peeling. Rev. Sci. Instrum. 2009, 80, 053104. [CrossRef]

38. Compton, R.N.; Miller, J.C.; Carter, A.E.; Kruit, P. Resonantly Enhanced Multiphoton Ionization of Xenon: Photoelectron Energy Analysis. Chem. Phys. Lett. 1980, 71, 87-90. [CrossRef]

39. Neese, F. The ORCA Program System. Wiley Interdiscip. Rev. Comput. Mol. Sci. 2012, 2, 73-78. [CrossRef]

40. Ekström, U.; Visscher, L.; Bast, R.; Thorvaldsen, A.J.; Ruud, K. Arbitrary-Order Density Functional Response Theory from Automatic Differentiation. J. Chem. Theory Comput. 2010, 6, 1971-1980. [CrossRef]

41. Weigend, F. Accurate Coulomb-Fitting Basis Sets for H to Rn. Phys. Chem. Chem. Phys. 2006, 8, 1057-1065. [CrossRef] [PubMed]

42. Krishnan, R.; Binkley, J.S.; Seeger, R.; Pople, J.A. Self-Consistent Molecular Orbital Methods. XX. A Basis Set for Correlated Wave Functions. J. Chem. Phys. 1980, 72, 650-654. [CrossRef] 
43. Frisch, M.J.; Pople, J.A.; Binkley, J.S. Self-Consistent Molecular Orbital Methods 25. Supplementary Functions for Gaussian Basis Sets. J. Chem. Phys. 1984, 80, 3265-3269. [CrossRef]

44. De Souza, B.; Farias, G.; Neese, F.; Izsák, R. Predicting Phosphorescence Rates of Light Organic Molecules Using Time-Dependent Density Functional Theory and the Path Integral Approach to Dynamics. J. Chem. Theory Comput. 2019, 15, 1896-1904. [CrossRef] 